\title{
EN BUSCA DEL ORIGEN DEL CONOCIMIENTO: EL DILEMA DE LA REALIDAD
}

\author{
IN SEARCH OF THE ORIGIN OF KNOWLEDGE: THE DILEMMA OF REALITY
}

\author{
Raúl Omar Di Marco-Morales*
}

\begin{abstract}
RESUMEN
El artículo muestra que no es raro tener la realidad como objetivo primordial del conocimiento, el cual se debe centrar en el progreso del hombre por medio de la investigación científica. El conocer y el saber algo (dilema que reside en los seres humanos) se está convirtiendo en un problema en su diario vivir. El conocimiento es un organismo con vida, ya que cambia y se enriquece con las nuevas experiencias, de modo tal que puede pasar de un estado inicial a un estado final más complejo y más eficaz, gracias a las principales características del conocimiento científico o la nueva interpretación sobre algún tema. Cada hombre de ciencia tiene un concepto de verdad diferente, ya que han explicado el conocimiento también en formas distintas y han definido la teoría científica como una metáfora, considerando que la ciencia es un conocimiento de lo unitario, mientras que las opiniones son simplemente de la multitud.
\end{abstract}

Palabras Clave: Epistemología, Características del conocimiento, Revolución científica, Paradigmas, Metáforas.

\begin{abstract}
This article shows that it is not rare to have as its primary objective reality of knowledge, which must focus on human progress through scientific research. Knowing and knowing something (dilemma resides in humans) is becoming a problem in their daily lives. Knowledge is an organism with life as it changes and is enriched with new experiences, so that can go from an initial state to a more complex and more efficient final state, thanks to the main features of the new scientific knowledge or interpretation on any issue. Every scientist has a different concept of truth, as explained knowledge in various forms and also defined scientific theory as a metaphor, considering that science is a knowledge of the unit, while the opinions are merely from the crowd.
\end{abstract}

Keywords: Epistemology, Characteristics Knowledge, Scientific Revolution, Paradigms, Metaphors.

Fecha de recepción: Julio 17 de 2015 / Fecha de aceptación: Septiembre 08 de 2015

Tipología: Artículo de Reflexión

Para citar el artículo: Di Marco, M. R. (2015). En busca del origen del conocimiento: El dilema de la realidad. Praxis. Vol. 11,150 - 162

*Ingeniero Civil (UniAndes), Especialista en Finanzas (UNAB), Magister en Administración (USTA), Candidato a Doctor en Administración (Universidad Nacional de Rosario-Argentina). Docente de la Universidad Libre de Colombia, Seccional Socorro. Colombia. Email: raulomard@yahoo.com 


\section{INTRODUCCION}

$\mathrm{H}$ e de decir que no conocía mucho sobre el significado de la palabra epistemología, pero tenía presente que el conocer surge como indica Sabino (1980, p.12) "ligado a la práctica vital y al trabajo de los hombres", por lo que lo hace ser en la actualidad una tarea esencial del hombre; de ahí, el conocimiento es más que nada un proceso y que la ciencia debe ser vista como una de las actividades que el hombre realiza dirigida y enmarcada hacia el desarrollo de la sociedad.

No es importante el nivel social en que se encuentre el hombre, toda persona o todo grupo ya que se hallan en actitud de conocer; pero al ser múltiples las circunstancias que rodean el conocimiento, los tipos de conocimientos que adquiere el hombre tienden a ser también diferentes, como lo es el tipo espontáneo o de sentido común: el que se adquiere (sin estar sometido a un método) por el sólo hecho de vivir y de estar en contacto con las cosas (saber básico); el saber crítico (que sigue un método con una coherencia lógica) como el del filósofo y el científico; el saber técnico (saber en dependencia con ciertas estructuras sociales). De ahí, no todos hacen caso omiso de las condiciones sociales en que están involucrados los procesos cognoscitivos, como lo indica Bunge (1972) al decir que: "la ciencia como actividad -como investigación- pertenece a la vida social” (p.9), y Sabino (1980) indicando que el conocimiento no es sólo la relación entre sujeto y objeto, ya que "se desenvuelve dentro de las ciencias sociales y de la conducta” (p. 26).

El objetivo básico del conocimiento es descifrar la realidad, pero de fondo, el target de la investigación científica es el progreso del hombre. Una investigación es una serie de actividades que se deben desarrollar dentro de proceso metódico, como son: de planteamiento del problema, de formulación de hipótesis, la construcción de la matriz de datos, de sus características y hasta los propósitos que se persiguen, que finalmente han de dejar un material que servirá como materia prima para nuevas investigaciones; Samaja
(2010) afirma: “el proceso de investigación se refiere a la totalidad de los componentes, dimensiones y momentos de la investigación científica"(p. 206).

Sabino (1980) refiere que el conocer y el saber algo que se requiere para desarrollar la vida, es un problema que radica fundamentalmente en los seres humanos que buscan responder a sus propias inquietudes; pero, de igual manera, Sabino (1980) afirma que la verdad no se muestra como tal, que debe ser buscada e indagada por medio de un trabajo de investigación (p. 11).

De ahí, que la investigación científica debe ser concebida como un proceso mediante el cual un sujeto que se inserta necesariamente en un cierto contexto, con el tiempo alcanzará un producto (un conocimiento más objetivo y satisfactorio) verificable sobre los hechos que nos rodean. Samaja (2010) sitúa al conocimiento científico como uno de los modos posibles de conocimiento humano, siempre y cuando lo que se obtiene sea un producto de un proceso de investigación científica. Por tal motivo es importante, hablar sobre la teoría del conocimiento como tal (p. 29).

\section{Teoría del conocimiento}

Si se quisiera problematizar alguna cuestión desde el punto de vista epistemológico y en especial a lo que se refiere a la metodología de la investigación, se revisarán algunos de los problemas del conocimiento y del origen del mismo, se deben comentar las principales características del conocimiento científico.

Surge entonces el primer interrogante: ies posible tener algún conocimiento de la realidad? Lo que se quiere llegar a saber es si es posible llegar al conocimiento, es decir, si el sujeto puede o no aprender del objeto y por ende llegar a una adecuada representación de la realidad, o simplemente, el hombre no puede tener ninguna seguridad respecto del conocimiento de las cosas; Hessen (2002) trata de resolver esta dificultad planteando unas posturas como se muestran en el Tabla 1, buscando esclarecer el problema. 
Tabla 1

Posibilidad del Conocimiento

\begin{tabular}{|c|c|}
\hline \multirow{3}{*}{ AGNOSTICISMO } & $\begin{array}{l}\text { Postura radical que afirma que es imposible conocer. Su } \\
\text { principal representante es Gorgias de Leontini (484-374 }\end{array}$ \\
\hline & $\begin{array}{l}\text { A.C.): el cual expresó: "Nada existe y si algo existe no es } \\
\text { cognoscible por el hombre". Lo que muestra es una }\end{array}$ \\
\hline & contradicción lógica. \\
\hline \multirow{3}{*}{ ESCEPTICISMO } & $\begin{array}{l}\text { Postura moderada que afirma no saber si es posible conocer; } \\
\text { aparece con Pirrón (365-270 A.C.): "Las cosas no se pueden }\end{array}$ \\
\hline & $\begin{array}{l}\text { conocer, la duda es la única forma de sabiduría”. El sujeto no } \\
\text { puede aprender el objeto. Los sentidos nos engañan. }\end{array}$ \\
\hline & $\begin{array}{l}\text { El hombre comete muchos errores y hay diversidad de opi- } \\
\text { niones respecto de una misma cosa. }\end{array}$ \\
\hline \multirow{3}{*}{ DOGMATISMO } & $\begin{array}{l}\text { Postura que reconoce la posibilidad del conocimiento; } \\
\text { Pitágoras (585-500 A.C.): "El punto es la unidad que tiene }\end{array}$ \\
\hline & $\begin{array}{l}\text { una posición". Existe una confianza total en el poder de la } \\
\text { razón para conocer. Ante esta verdad, la comunidad y en }\end{array}$ \\
\hline & $\begin{array}{l}\text { especial sus integrantes, pueden asumir alguna de estas tres } \\
\text { posiciones: ignorancia, búsqueda o sabiduría. }\end{array}$ \\
\hline \multirow{3}{*}{ RELATIVISMO } & $\begin{array}{l}\text { Postura que reconoce que el conocimiento sí es posible, dado } \\
\text { que podemos tener algún grado de certeza, pero }\end{array}$ \\
\hline & $\begin{array}{l}\text { rechaza que el conocimiento sea absoluto, ya que indica que } \\
\text { el conocimiento es sólo producto de la experiencia y }\end{array}$ \\
\hline & $\begin{array}{l}\text { esta es relativa, por lo cual no hay verdades universales ni } \\
\text { absolutas. Pitágoras y Crítias fueron sus representantes. }\end{array}$ \\
\hline \multirow{3}{*}{ CRITICISMO } & $\begin{array}{l}\text { Postura que acepta que el hombre pueda llegar a conocer y } \\
\text { por ende a poseer la verdad, llegando a tener los }\end{array}$ \\
\hline & $\begin{array}{l}\text { conocimientos que le den certeza, sin embargo, se hace in- } \\
\text { dispensable justificar racionalmente la forma como se }\end{array}$ \\
\hline & $\begin{array}{l}\text { llega al conocimiento; Aristóteles, Descartes y Kant, fueron } \\
\text { sus principales representantes. }\end{array}$ \\
\hline \multirow{3}{*}{ PRAGMATISMO } & $\begin{array}{l}\text { Postura que acepta la posibilidad del conocimiento, que gra- } \\
\text { cias a la interacción del sujeto objeto se convierte }\end{array}$ \\
\hline & $\begin{array}{l}\text { en fundamento de la verdad y de certeza; la verdad es práctica } \\
\text { y se confunde con lo útil y valioso. }\end{array}$ \\
\hline & $\begin{array}{l}\text { Nietzsche, James, Simmel y Dewey, fueron sus principales } \\
\text { exponentes. }\end{array}$ \\
\hline
\end{tabular}

Fuente: Elaboración propia basada en Hessen, de su libro: Teoría del Conocimiento, 2002. 
Si se quisiera tratar el tema del origen del conocimiento, surge el segundo interrogante: ¿el conocimiento se origina por la experiencia o por la razón?, es decir, si el hombre viene de por sí provisto de ciertos conocimientos o requiere adquirirlos en el transcurrir del desarrollo de sus propias facultades sensibles e intelectivas a la vez. Hessen (2002) plantea algunas fuentes (ver Tabla 2), los cuales buscan darle solución al problema del origen del conocimiento (p. 24).

\section{Tabla 2}

Origen del Conocimiento

\begin{tabular}{|c|c|}
\hline \multirow{4}{*}{ RACIONALISMO } & $\begin{array}{l}\text { El conocimiento tiene su origen en la razón (la experiencia } \\
\text { no cuenta). Descartes en su libro El discurso del }\end{array}$ \\
\hline & $\begin{array}{l}\text { Método indica que es indispensable tomar una actitud radical } \\
\text { frente a todo lo que uno da por aceptado; se duda }\end{array}$ \\
\hline & $\begin{array}{l}\text { de la existencia de todo, lo que lleva a deducir su gran prin- } \\
\text { cipio: "Pienso, luego existo", haciendo derivar del pensar su }\end{array}$ \\
\hline & $\begin{array}{l}\text { existencia. Aquí prima el sujeto frente al objeto. Leibnitz, } \\
\text { desea que todo sea claro y demostrable por la razón. }\end{array}$ \\
\hline \multirow{4}{*}{ EMPIRISMO } & $\begin{array}{l}\text { El origen está en la experiencia, ya que todo lo que tenga que } \\
\text { ver con el pensamiento inevitablemente ha tenido }\end{array}$ \\
\hline & $\begin{array}{l}\text { que pasar por los sentidos; la objetividad del mundo se de- } \\
\text { muestra a través de las sensaciones externas. }\end{array}$ \\
\hline & $\begin{array}{l}\text { Berkeley sostiene que el conocimiento se reduce a un con- } \\
\text { junto de impresiones o sensaciones o ideas, que proceden }\end{array}$ \\
\hline & de una sustancia espiritual: Dios. \\
\hline \multirow{3}{*}{$\begin{array}{l}\text { I N T E L E C T U A } \\
\text { LISMO }\end{array}$} & $\begin{array}{l}\text { Busca una integración al sostener que en todo conocimiento, } \\
\text { tanto la razón como los sentidos desempeñan una }\end{array}$ \\
\hline & $\begin{array}{l}\text { función necesaria. Aristóteles formuló esta teoría al sostener } \\
\text { que las ideas son conceptos mentales con }\end{array}$ \\
\hline & $\begin{array}{l}\text { fundamento en lo real; además conocer científicamente es } \\
\text { conocer las cosas por sus causas. }\end{array}$ \\
\hline \multirow{4}{*}{ APRIORISMO } & $\begin{array}{l}\text { El conocimiento es un compendio entre los datos proporcio- } \\
\text { nados por la experiencia y la estructura del sujeto. }\end{array}$ \\
\hline & $\begin{array}{l}\text { Kant, que es su representante, consideraba que el raciona- } \\
\text { lismo llegaba a verdades universales y necesarias pero que }\end{array}$ \\
\hline & $\begin{array}{l}\text { no sabía explicar la relación de esas verdades con la expe- } \\
\text { riencia. En su obra La crítica de la razón pura pone de }\end{array}$ \\
\hline & $\begin{array}{l}\text { manifiesto que se debe hacer un análisis exhaustivo de la } \\
\text { facultad de la razón, determinando sus fuentes y límites. }\end{array}$ \\
\hline
\end{tabular}

Fuente: Elaboración propia basada en Hessen, de su libro: Teoría del Conocimiento, 2002. 
Barragán (1977) menciona que Popper afirmó que no hay fuentes últimas de conocimiento, por lo cual debe darse la bienvenida a toda fuente y a toda sugerencia, las cuales deben ser sometidas a un examen crítico ( $\mathrm{p}$. 48); además indicó que Popper es partidario de considerar el conocimiento como un desarrollo progresivo, lo que viene a confirmar que el avance del conocimiento consiste principalmente en la modificación de lo existente o del conocimiento precedente, pero Barragán (1977) afirma que la intuición intelectual y la imaginación llegan a tener un papel primordial, pero que no son confiables ya que pueden conducir al error.

En la construcción de cualquier marco teórico hay que precisar que un proceso de investigación no es más que una continuidad de momentos metodológicos ó fases, que son posibles de distinguir a medida que se van moldeando, dentro de un camino de carácter dinámico y procesal (no lineal) que deberá recorrer el investigador; el conocimiento es un organismo en crecimiento, de ahí que cambie y se enriquezca con las nuevas experiencias, de modo tal que pueda pasar de un estado de menor conocimiento (inicial) a un estado más complejo y más eficaz (final), donde el trabajo de cada pesquisa o exploración en particular es parte de un esfuerzo continuo y mucho mayor en el desarrollo de nuevos hallazgos. Puledda (1996) menciona que para Marx "el trabajo no es solamente una actividad práctica, sino también una categoría del conocimiento y es lo que constituye justamente la vida y la conciencia de la especie” (p. 54).

Hablar de la esencia del conocimiento es plantear el tercer interrogante: ¿cuáles son las características del conocimiento científico? En la interacción sujeto-objeto se ha entendido que el intelecto es una actividad del sujeto, por la cual pretende captar el sentido del objeto; de ahí que Bunge (1972) describa las principales características del conocimiento científico y que se resumen en el Tabla 3 y 4, donde sobresale que el conocimiento científico es fáctico, ya que parte de los hechos, descarta hechos, produce nuevos hechos y los explica (p. 16-36).

Además, complementando a Bunge (1972) que indica que: "la ciencia es valiosa como una herramienta para domar la naturaleza y remodelar la sociedad"(p. 36), Ynoub (2012) confirma que con mucha frecuencia los hallazgos que se van presentando pueden inspirar nuevos interrogantes, por lo que hay que buscar nuevas respuestas, ya que toda interpretación de datos puede ofrecer nuevos caminos a recorrer y nuevas lecturas a realizar, ya que da paso a nuevos objetivos e hipótesis a los que se les deben dar respuestas (p. 34). Por otro lado, De la Maza y Villanueva (2011) afirman que la "ciencia es una palabra increíblemente malentendida" (p. 22), pero encuentran de gran relevancia la importancia del estado actual del conocimiento e indican que es la base para las teorías científicas e investigaciones a futuro. 
Tabla 3

Características del Conocimiento Científico

\begin{tabular}{|c|c|}
\hline CARACTERISTICAS & OBSERVACIONES \\
\hline El conocimiento científico es fáctico & $\begin{array}{l}\text { Parte de los hechos, los descarta, produce } \\
\text { nuevos hechos y los explica. La ciencia } \\
\text { intenta describir los hechos tal como son, } \\
\text { independientemente de su valor emocional. }\end{array}$ \\
\hline $\begin{array}{l}\text { El conocimiento científico va más } \\
\text { allá de los hechos }\end{array}$ & $\begin{array}{l}\text { Trasciende los hechos, ya que no se limita } \\
\text { a los hechos observados ni percibidos; se } \\
\text { pueden producir nuevos hechos, los que se } \\
\text { pueden explicar y difundir. }\end{array}$ \\
\hline La ciencia es analítica & $\begin{array}{l}\text { La Investigación científica trata de entender } \\
\text { toda circunstancia en términos de sus fac- } \\
\text { tores; intenta descubrir los elementos y las } \\
\text { interconexiones que la componen. }\end{array}$ \\
\hline $\begin{array}{l}\text { La investigación científica es } \\
\text { especializada }\end{array}$ & $\begin{array}{l}\text { La especialización tiende a estrechar la vi- } \\
\text { sión del científico. }\end{array}$ \\
\hline $\begin{array}{l}\text { El conocimiento científico es trans- } \\
\text { parente, claro y preciso }\end{array}$ & $\begin{array}{l}\text { Mientras el conocimiento ordinario es du- } \\
\text { doso y desacertado, en el conocimiento } \\
\text { científico se obtiene claridad y precisión, } \\
\text { ya que los problemas se formulan en forma } \\
\text { clara y sus resultados son precisos. }\end{array}$ \\
\hline $\begin{array}{l}\text { El conocimiento científico es } \\
\text { comunicable }\end{array}$ & $\begin{array}{l}\text { El conocimiento no es privado, es público, } \\
\text { no es inefable sino expresable, ya que los } \\
\text { científicos consideran el secreto como ene- } \\
\text { migo del progreso o de la ciencia. }\end{array}$ \\
\hline $\begin{array}{l}\text { El conocimiento científico es } \\
\text { verificable }\end{array}$ & $\begin{array}{l}\text { Las suposiciones deben ser puestas a com- } \\
\text { probación, por lo que deben aprobar el } \\
\text { examen de la experiencia. }\end{array}$ \\
\hline $\begin{array}{l}\text { La investigación científica es } \\
\text { metódica. }\end{array}$ & $\begin{array}{l}\text { La investigación debe ser planeada, no } \\
\text { errática; la ciencia emplea el método expe- } \\
\text { rimental, el cual requiere de un proceso, de } \\
\text { observación y del registro de los fenómenos, } \\
\text { exigiendo el análisis de los datos obtenidos } \\
\text { en busca de la verdad. }\end{array}$ \\
\hline
\end{tabular}

Fuente: Elaboración propia basada en Bunge, de su libro: El proceso de investigación, 1972.

Samaja (2010) expresa "que un conocimiento que egresa del proceso de investigación, inmediatamente se transforma en condiciones de investigación para nuevos procesos, y si no es incorporado se vuelve alimento de roedores e insectos y no un hecho de la cultura científica” (p. 47). Por tal motivo, el conocimiento o la nueva interpretación sobre algún tema, abre nuevas preguntas y nuevas conjeturas, las que llevan a cimentar las bases para su abordaje e inicio de un nuevo ciclo de investigación. 
Tabla 4

Otras características del Conocimiento Científico

\begin{tabular}{|l|l|}
\hline \multicolumn{1}{|c|}{ CARACTERISTICAS } & \multicolumn{1}{c|}{ OBSERVACIONES } \\
\hline $\begin{array}{l}\text { El conocimiento científico es } \\
\text { metódico }\end{array}$ & $\begin{array}{l}\text { Una ciencia es un camino de ideas conectadas ló- } \\
\text { gicamente entre sí. }\end{array}$ \\
\hline $\begin{array}{l}\text { El conocimiento científico } \\
\text { es general }\end{array}$ & $\begin{array}{l}\text { El científico se ocupa del hecho singular en la medi- } \\
\text { da en que éste es miembro de una clase mayor, con } \\
\text { lo que ubica hechos singulares en pautas generales. }\end{array}$ \\
\hline $\begin{array}{l}\text { El conocimiento científico } \\
\text { es legal }\end{array}$ & $\begin{array}{l}\text { El conocimiento científico implanta los hechos parti- } \\
\text { culares en pautas generales llamadas leyes sociales, } \\
\text { por lo que intenta llegar al origen de las cosas. }\end{array}$ \\
\hline La ciencia es explicativa & $\begin{array}{l}\text { Trata o intenta explicar los hechos en términos de } \\
\text { reglas, mientras éstas en términos de causas. Los } \\
\text { investigadores intentan responder al porqué. }\end{array}$ \\
\hline $\begin{array}{l}\text { El conocimiento científico es } \\
\text { predictivo }\end{array}$ & $\begin{array}{l}\text { La suposición científica se caracteriza por su perfec- } \\
\text { tibilidad antes que por su certeza, ya que se funda } \\
\text { en leyes y sobre informaciones específicas evidentes } \\
\text { y relativas al estado de las cosas. }\end{array}$ \\
\hline La ciencia es libre y abierta & $\begin{array}{l}\text { El conocimiento científico no reconoce barreras } \\
\text { a priori que la limiten, haciéndola como un orga- } \\
\text { nismo en crecimiento, que mientras esté vivo va } \\
\text { caminando sin parar. }\end{array}$ \\
\hline La ciencia es útil & $\begin{array}{l}\text { La ciencia busca la verdad sin proponerse necesa- } \\
\text { riamente alcanzar resultados aplicables, ya sea a } \\
\text { corto, mediano o largo plazo. }\end{array}$ \\
\hline
\end{tabular}

Fuente: Elaboración propia basada en Bunge, de su libro: El proceso de investigación, 1972.

Para Kuhn (1989) el término ciencia requiere de un quehacer, de una actividad de las comunidades científicas que son sus mismos protagonistas; además, que la ciencia es un paradigma del conocimiento, de la racionalidad y del progreso (p. 50).

Finalmente, en la construcción de este marco teórico hay que precisar un planteamiento epistemológico en búsqueda de la verdad, en aquello que no está correcto y que afecta la dignidad del individuo, para trascender a la sociedad.

\section{Objetividad del conocimiento}

Samaja (2010) comenta que la epistemología es la filosofía de la ciencia que requiere de ciertos saberes previos acerca de su naturaleza y sus funciones; de ahí que estudie los problemas relacionados con el conocimiento propiamente científico, siendo un estudio crítico de los principios, de las hipótesis y de los resultados de las diversas ciencias, destinado a determinar su origen lógico, su valor y su contenido. Bautista (2011) indica que la 
palabra epistemología proviene de dos vocablos: "episteme" que significa conocimiento científico y "logos" que significa tratado (p. 27). Dado que la ciencia es una forma de conocimiento, epistemología se podría llevar al contexto del conocimiento de la ciencia o simplemente como la teoría de la ciencia.

Barragán (1977) menciona que desde tiempos inmemorables, todo conocimiento verdadero debía tener una disposición no en las apariencias de las cosas, sino en un fundamento consistente al cual sólo tenía acceso la razón (p. 72). Además indica que Parménides entiende que la verdad no puede ser otra cosa que el desvelamiento del ser, mientras que Heráclito estaba convencido de que la verdad sólo se descubre a través de la razón. De ahí, que Barragán (1977) mencioné que uno de los primeros filósofos griegos en tratar de definir la episteme (ciencia) fue Platón, el cual la coloca como el conocimiento de la realidad tal como es o el fundamento de la realidad, el conocimiento fundado y que revela la existencia de lo que verdaderamente es, al que él llamó "el bien”; además, Platón muestra que la diferencia radica en la doxa (opinión), a la cual define como un tipo de saber que no posee fundamento (no tiene rigurosa reflexión crítica), pero que es un conocimiento particular, cambiante y vulgar del hombre, dependiendo de los sentidos.

Platón definía al filósofo como aquel que ama la episteme con pasión, ya que es el mismo filósofo, un hombre que tiene el verdadero conocimiento. Aristóteles que es mencionado por Barragán (1977) afirmó que conocer las cosas en sus causas y en sus esencias es conocerlas en su aspecto de universalidad y de necesidad. Barragán (1977) habla de Santo Tomás, el cual concibe la verdad como la adecuación del pensamiento con las cosas, mostrando que las cosas en sí, tienen su propia verdad y entidad: verdad ontológica (p. 75).

En la edad moderna, aparece Descartes que establece un fundamento distinto de la verdad con lo que queda reducida la verdad ontológica a la claridad con que se presenten las ideas; la verdad no depende del ser real, sino de la claridad con que se presenten las ideas del ser en nuestras mentes; sin embargo, Samaja (2010) menciona que La crítica del juicio escrito por Kant, "contiene el último tramo del puente que Kant intentó tender entre la realidad y el sujeto humano" (p. 74), lo que lleva a que no se pueda encontrar un criterio universal de la verdad por vía de los fenómenos de experiencia de la materia.

Además, Barragán (1977) señala a Hegel como el que identifica el ser con el conocer y lo racional con lo real. Menciona que para Hegel la verdad queda identificada con el proceso racional y es identidad del pensamiento consigo mismo, generando que la verdad, que también está en movimiento, deje de ser una simple conformidad para ser concebida como una identidad; finalmente Barragán (1977) nombra a Heidegger, el cual indica que la verdad no es otra cosa que el desvelamiento, el desocultamiento, la presentación y la apertura del ser a la inteligencia del hombre; de ahí que la verdad no se pueda reducir al acto del juicio que hace el entendimiento.

Como se muestra, cada hombre de ciencia tiene un concepto de verdad diferente debido a que han explicado el conocimiento también en forma diferente. A quienes dan más importancia al sujeto (subjetivismos, racionalismos, idealismos) no pueden ver la verdad sino desde el punto de vista de la razón, del sujeto. Savater (1999) dice que: "la razón es un servicio público intelectual: un ómnibus” (p. 9), debido a que no exige nada especial para funcionar y que sólo pide ser usada. Por otro lado están los que le dan más importancia al objeto, indicando que el conocimiento es una relación entre sujeto-objeto, mientras la verdad es una adecuación entre el sujeto y el objeto (Samaja, 2010).

\section{Revoluciones científicas, paradigmas y metáforas}

Balmes (1987) afirma que el hombre desgraciadamente no siempre encuentra lo que necesita y es demasiado ansioso para esperar que los siglos que él no ha de ver proporcionen a las generaciones futuras las revelaciones deseadas, por eso finge y en vez de construir sobre la realidad, va a edificar sobre sus propias creaciones de su pensamiento (p. 58). 
Kuhn (1989) indica en su libro ¿Qué son las revoluciones científicas? que la práctica científica implica siempre la producción y explicación de generalizaciones sobre la naturaleza, las cuales, presuponen un lenguaje que lleva consigo conocimiento de la misma, por lo que la característica esencial de las revoluciones científicas es la alteración del conocimiento de la naturaleza intrínseco al lenguaje mismo. Kuhn (1989) además muestra que estos cambios revolucionarios son en un sentido holistas, ya que no pueden hacerse poco a poco, afirmando que la mayoría del avance científico es de tipo normal acumulativo, pero los cambios revolucionarios son diferentes y más problemáticos ya que ponen en juego descubrimientos que no pueden acomodarse dentro de los conceptos habituales anteriores a los hallazgos (p. 72).

Sin embargo, Kuhn (1998) en su libro La estructura de las revoluciones científicas, establece una definición para paradigma: "es un modelo o patrón aceptado” (p. 51), viniendo a desempeñar un rol: vehículo para la teoría científica. Kuhn (1998) muestra que la investigación científica normal va dirigida a la articulación de aquellos fenómenos y teorías que ya proporciona el paradigma, como la visión completa de la realidad o forma de verla; de ahí surge una de las implicaciones más importantes del trabajo de Kuhn, la identificación de paradigmas como realidades alternativas, denotando una visión implícita o explícita de la realidad.

El trabajo, bajo el paradigma (indica Kuhn), no puede llevarse a cabo en ninguna otra forma y la deserción del paradigma significa: dejar de practicar la ciencia que la define; por tal motivo, la determinación del hecho significativo, el acoplamiento de los hechos con la teoría y la articulación con la teoría, son los pilares sobre los que apoyan las revoluciones científicas, ya que rompen con una tradición de práctica científica e introduce otra nueva que se lleva a cabo con reglas diferentes y dentro de un universo de razonamiento igualmente diferente.

De ahí, que las revoluciones científicas se consideren como aquellos episodios de desarrollo en que un antiguo paradigma es reemplazado, ya sea completamente o por partes, por uno nuevo e incompatible. Es así, que se llama revolución a un cambio de paradigma debido a que un paradigma existente ha dejado de funcionar adecuadamente en la exploración de un aspecto de la naturaleza.

Kuhn (1998) argumenta que la asimilación de todas las nuevas teorías ha exigido la destrucción de un paradigma anterior, llevando a un conflicto (revolución) entre las escuelas competitivas de pensamiento científico.

Para Kuhn (1998) hay tres tipos de fenómenos para la investigación científica general y fundamentada, los cuales no son permanentes; ellos son:

1. Los fenómenos que ya han sido bien justificados por los paradigmas existentes y que ha mostrado que son reveladores propios de la naturaleza de las cosas

2. Los fenómenos cuya esencia es señalada por paradigmas existentes, pero cuyos detalles sólo pueden ceñirse a través de una coyuntura contigua a la teoría.

3. Los fenómenos (incoherencias reconocidas) cuyo rasgo característico es la prohibición total a ser asimilados en los paradigmas existentes, por lo que se producen nacientes teorías (p. 155)

La aplicación del término paradigma en el ámbito de la reflexión epistemológica se debe a Thomas Kunh, menciona Ynoub (2012), e indica que sin la aceptación de un paradigma, no habría ciencia normal por la falta de enigmas que no hayan sido todavía resueltos. Para Kuhn (1998) y como resultado de ello, la recepción de un nuevo paradigma requiere de una redefinición de la ciencia correspondiente, de ahí que la tradición científica que surge de una revolución científica es realmente único e inigualable con respecto a la que existía con anterioridad (p. 166).

Por otro lado, el rol de los paradigmas como formas de ver la realidad y que determinan una mentalidad y una ideología, viniendo a regir la concepción de la ciencia (García y Giacobbe, 2009), se podrían subdividir en cuatro formas de 
ver el mundo: el funcionalista, el interpretativo, el humanista-radical y el estructuralista-radical, reflejando un entorno metateórico sobre la naturaleza de la ciencia, la naturaleza de la sociedad y la dimensión objetiva-subjetiva.

Al estudiar un paradigma, Kuhn (1998) afirma que el científico adquiere al mismo tiempo tres elementos básicos: teoría, método y normas, que se constituyen como inseparables al paradigma; cuando estos cambian (los paradigmas), hay una transformación importante de los criterios que determina la legitimidad tanto de los problemas como de las soluciones propuestas, por lo que se podría decir: que los paradigmas son parte constitutiva de la ciencia (p. 174). Por consiguiente, Kuhn (1998) indica que en tiempo de revolución es cuando la tradición científica cambia, el conocimiento que el científico tiene debe ser reevaluado (p. 177). Aunque el mundo no varía con una reestructuración de los paradigmas, el científico empieza a trabajar en un mundo diferente a sus sentidos y a su razón; sin embargo, el científico que acepta un nuevo paradigma, dice Kuhn (1998) es: "como el hombre que lleva lentes inversores”, ya que dado un nuevo paradigma, la interpretación de datos es crucial para explorarlo (p. 191).

Es así, dice Kuhn (1998), que los científicos hablan con frecuencia de "las vendas que se les caen de los ojos" o de la "iluminación repentina" que inunda un enigma inicialmente incierto; ningún sentido se ajusta a esos centelleos de la intuición (anómala o congruente) que dan paso al nacimiento de un nuevo paradigma.

De esta manera estas metáforas cumplen un papel fundamental en las revoluciones científicas, ya que implican un cambio fundamental de modelo, un cambio de la noción de qué es semejante y que es diferente a qué, llevando a una recategorización del mundo al generar un nuevo tipo de similitudes y haciendo surgir nuevos significados.

La metáfora usualmente se ve como un componente literario y descriptivo, pero según Palma (2004), fundamentalmente es una forma creativa que procede mediante afirmaciones que actúan como generadores de un nuevo significado; en un trabajo de investigación, indica Ynoub (2012), los científicos ven el mundo metafóricamente debido al lenguaje y los conceptos que filtran y estructuran las percepciones de su tema de estudio, es decir, utilizan la metáfora para generar y proveer la base para la investigación científica.

Hay diferentes tipos de estructuras conceptuales metafóricas, que se pueden observar en la Tabla 5; según Lakoff y Johnson (1991) la tradición literaria indica que la metáfora puede manifestarse en todo tipo de elementos gramaticales y una vez configurada, va a contribuir a canalizar la comprensión de un determinado discurso; además, que la metáfora está embebida en la vida cotidiana, no solamente en el dialecto, sino también en el intelecto y en la acción (p. 23). Por otro lado, Palma (2004) indica que la expresión metafórica funciona como un sustituto de una expresión literal en forma de lenguaje figurado, cuya función es la analogía o semejanza a su equivalente literal y para que funcione, el destinatario debe manejar el lenguaje metafórico (p. 22). 
Tabla 5

Tipos de estructuras conceptuales metafóricas

\begin{tabular}{|l|l|}
\hline \multicolumn{1}{|c|}{ ESTRUCTURA } & \multicolumn{1}{c|}{ DEFINICION } \\
\hline METAFORAS ESTRUCTURALES & $\begin{array}{l}\text { Son aquellas cuando se dice que un concepto } \\
\text { está estructurado metafóricamente en otro y } \\
\text { que puede ser extendido de ciertas maneras } \\
\text { pero no de otras; las nociones no emergen } \\
\text { directamente sólo de la experiencia, por lo } \\
\text { que deben ser estructurados a partir de las } \\
\text { metáforas normativas dominantes. Ejemplo: } \\
\text { el tiempo es oro. }\end{array}$ \\
\hline METAFORAS ORIENTACIONALES & $\begin{array}{l}\text { Son las que dan a un concepto y una orienta- } \\
\text { ción especial, organizando un sistema global } \\
\text { de nociones con relación a otros; tienen que } \\
\text { ver con la orientación espacial y pueden } \\
\text { haber surgido de nuestra experiencia física y } \\
\text { social. Ejemplo: lo racional es lo principal, lo } \\
\text { emotivo es lo secundario. }\end{array}$ \\
\hline METAFORAS ONTOLOGICAS & $\begin{array}{l}\text { Son las que surgen directamente de nuestra } \\
\text { propia experiencia y que son necesarias para } \\
\text { tratar de enfrentarnos de manera racional a } \\
\text { ellas, caracterizando un fenómeno de forma } \\
\text { particular mediante su apreciación como una } \\
\text { entidad, una sustancia, una persona; sirven } \\
\text { a efectos diversos ya que proporcionan la } \\
\text { base para una variedad extraordinariamente } \\
\text { amplia de metáforas. Ejemplo: la devaluación } \\
\text { me enferma. }\end{array}$ \\
\hline
\end{tabular}

Fuente: Elaboración propia basada en Lakoff y Johnson, de su libro: Metáforas de la vida diaria, 1991.

De esta manera, las metáforas permiten entender de forma sistemática un dominio de nuestra experiencia en términos de otro y así satisfacer la necesidad desapasionada de una explicación de la verdad, mediante una apropiada estructuración de la experiencia, sin dejar a un lado las expectativas del subjetivismo sobre el juicio personal del conocimiento, es decir, como dice Ynoub (2012): "la ciencia va detrás de conocimientos que develan regularidades necesarias para los fenómenos que investiga” (p. 25).

\section{CONCLUSIÓN}

El conocimiento es un proceso gradual y sistemático donde se van superando las primeras experiencias de los productos hasta llegar a un saber más acorde con la realidad, tanto con la naturaleza como la vida social e histórica de las comunidades, aceptando que el conocimiento de esa realidad tiene que ser un proceso dinámico y gradual, no es un hecho aislado, por lo que comparto completamente la afirmación de Mario Bunge (1972), 
al referirse que "la investigación se abre camino en la selva de los hechos, ya que en la ciencia no hay caminos reales" (p. 48).

Se ha comentado sobre las posibilidades, sus orígenes y las características del conocimiento científico en busca de entender la actividad humana y el saber y entender la realidad; de ahí parte que la comunidad científica construye sistemáticamente estructuras teóricas y las comprueba sin recurrir ni aceptar las explicaciones caprichosas de los fenómenos dadas de manera vaga, ya que la ciencia es fundamentalmente una actividad racional que va dirigida hacia un entendimiento de lo general en vez de hacia la explicación de sucesos individuales.

La advertencia sobre la naturaleza metafórica de la teoría de las organizaciones puede apoyar al entendimiento de los fenómenos organizacionales, rompiendo con la rígida división que se tiene entre lo que constituye arte y ciencia, ya que estatus epistemológico de la metáfora puede contribuir por medio de la sensibilización de los científicos al abordaje y desarrollo de métodos de investigación relevantes, a ser aplicados a casos empresariales.

Estoy muy de acuerdo con lo que indican Lakoff y Johnson, que definen una teoría científica como una metáfora, y considero que la ciencia es un conocimiento de lo unitario, mientras que lo múltiple es simplemente una opinión.

Encontré que para la mayoría de los epistemólogos contemporáneos, el objeto de las ciencias fácticas es la explicación de los hechos, mientras que los cambios en las reglas que rigen los problemas, los conceptos y las explicaciones, pueden transformar una ciencia. De ahí que se requieran de paradigmas para entender los enigmas que la ciencia normal muchas veces se le ha dificultado explicar.

Es necesario tener conciencia de que la filosofía, al igual que los lineamientos científicos, necesita innovarse, ya que se requiere valorar y dar solución a los problemas que se van presentando en todo proceso real; de esta manera, la filosofía deja de ser la ciencia que tiene la verdad y comienzan los filósofos y la comunidad científica a encaminarse en busca de una verdad insuperable, la cual no es posible encontrar. No se trata de una filosofía que no tuviera más que decir, sino lo que las ciencias dicen y comprueban; innumerables teorías científicas del pasado ya han sido reemplazadas por otras y seguramente estas tendrán el mismo destino, sin embargo considero que nunca se llegará a la verdad absoluta en ningún área de investigación.

Las ciencias sociales como la Sicología, la Sociología, la Educación y la Antropología, entre otras, como en las ciencias del medio ambiente, comienzan la investigación en una forma metódica y controlada, además de ser crucial ya que se caracteriza por aprobar o desechar hipótesis que traten de explicar los fenómenos del comportamiento del hombre; no cabe duda que su punto de partida está también en la observación de los hechos como en toda ciencia, seguido por la formulación de problemas y la estructuración de hipótesis, siempre en la búsqueda de pruebas para confrontar dichas hipótesis, con la finalidad de establecer una ley o norma explicativa de la conducta social de los individuos. Esta información es devuelta al problema original, por lo que, el problema se mantiene o se altera según lo dicten las pruebas por lo que se desata una revolución científica: el dilema de la realidad; después de esta revolución, la comunidad científica siempre trabajará en un mundo diferente y continuará con la exploración del nuevo paradigma que ha nacido.

Aunque Kuhn mencionaba que un investigador es un solucionador de enigmas y no un probador de los paradigmas establecidos, considero que durante la búsqueda de la solución de un enigma particular y sin poner a prueba al paradigma, se puede abordar el problema del dilema de la realidad ensayando una serie de métodos alternativos. Sin embargo, muchos de los instrumentos de recolección de la información, como son las entrevistas estructuradas (principal técnica utilizada de tipo cuantitativo) distan de la participación de los protagonistas, los cuales se limitan simplemente a responder cada pregunta y listo; 
pero, cuando se cambia la estrategia de abordaje como es el del tipo cualitativo por medio de las entrevistas a profundidad y las entrevistas focalizadas, promueven un acercamiento al conocimiento de una manera más natural utilizando los sujetos, lo cual viene a cambiar los papeles del investigador y el investigado, ya que el primero pasa a ser el neófito mientras el protagonismo se lo roba el entrevistado por ser el experto en comparación al investigador.

Por todo lo anterior, el conocimiento y la ciencia (dinámicas de naturaleza) siempre están en progreso.

\section{REFERENCIAS BIBLIOGRÁFICAS}

Balmes, J. (1987). El criterio. México. Editorial Porrúa. Barragan, H. (1977). Epistemología. Bogotá. Unión Gráfica Ltda.

Bautista, N. (2011). Proceso de la Investigación cualitativa. Epistemología, metodología y aplicaciones. Bogotá. Editorial Manual Moderno.

Bunge, M. 1958. LA CIENCIA, su método y su filosofía. Buenos Aires, Editorial Siglo Veinte.

De La Maza, C. y Villanueva, L. (2011). Diseño de investigación aplicado a las ciencias del medio ambiente. Santiago de Chile. Editorial Universitaria.
Hessen, J. (2002). Teoría del conocimiento. Bogotá. Momo Ediciones.

Garcia, J. y Giacobbe, M. (2009). Nuevos desafíos en investigación. Teorías, métodos, técnicas e instrumentos. Rosario. Homo Sapiens Ediciones.

Kuhn, T. (1989). ¿Qué son las revoluciones científicas? Y otros ensayos. Buenos Aires. Ediciones Paidós.

Kuhn, T. (1998). La estructura de las revoluciones científicas. D’vinni Editorial. Santafé de Bogotá. Fondo de cultura económica.

Lakoff, G. y Johnson, M. (1991). Metóforas de la vida cotidiana. Madrid. Ediciones Cátedra.

Palma, H. (2004). Metáforas en la evolución de las ciencias. Buenos Aires. Jorge Buadino Ediciones.

Puledda, S. (1996). Interpretaciones históricas del humanismo. Santiago de Chile. Ediciones Corfhu.

Sabino, C. (1980). El proceso de investigación. Santafé de Bogorá. El Cid Editor.

Samaja, J. (2010). Epistemología y metodología, elementos para una teoría de la investigación científica. Buenos Aires. Editorial Universitaria de Buenos Aires - Eudeba.

Savater, F. (1999). Las preguntas e la vida. Barcelona. Editorial Ariel, S.A.

Ynoub, R. (2012). Inédito I: La ciencia como práctica social: bases para situar el examen del proceso de investigación científica en sentido pleno. Buenos Aires. Inédito. p. 1 - 49.

Ynoub, R. (2012). El proyecto y la metodología de la investigación. Buenos Aires. Cengage Learning. 